\title{
MSFEM for the Linear 2D1D-Problem of Eddy Currents in Thin Iron Sheets *
}

\author{
Markus Schöbinger* Joachim Schöberl* Karl Hollaus * \\ * Institute for Analysis and Scientific Computing, \\ Technische Universität Wien, Vienna, Austria \\ (e-mail: markus.schoebinger@tuwien.ac.at).
}

Keywords: eddy current, multiscale finite element method, numerical model

\section{INTRODUCTION}

In rotating electrical machines, it is reasonable to assume that each iron sheet is exposed to the same field, thus it suffices to simulate only one sheet. In the case of the thickness of the sheet being small compared to the other dimensions, the three dimensional problem may be further reduced to a two dimensional one, coupled with a separate one dimensional problem in the direction of the thickness, which will be assumed to be the $z$ axis throughout this contribution. Examples of such an approach have been presented in Bottauscio and Chiampi (2002) and J. Pippuri and Arkkio (2010), where the coupling is realized via a nested iteration, and F. Henrotte and Geuzaine (2015), where this principle was used in the context of homogenization.

This contribution presents a novel approach to this idea utilizing a multiscale finite element method (MSFEM, Hollaus and Schöberl (to be published)). The main principle is to express the behavior of the solution along the $z$ axis via a polynomial ansatz which directly couples into the two dimensional problem, thereby eliminating the need to repeatedly solve two dependent problems. Such a method will be developed and tested for both the $A$ formulation and the $T$ formulation. All models assume a linear, timeharmonic setting.

\section{A FORMUlation}

In three dimensions, the weak form of the eddy current problem is given as: Find the magnetic vector potential $\mathbf{A} \in H($ curl $)$, satisfying suitable boundary conditions, so that

$$
\int_{\Omega} \mu^{-1} \operatorname{curl} \mathbf{A} \cdot \operatorname{curl} v+i \omega \sigma \mathbf{A} \cdot \mathbf{v} d \Omega=0
$$

for all test functions $\mathbf{v} \in H$ (curl). In (1) $\mu$ denotes the magnetic permeability, $i$ the imaginary unit, $\omega$ the angular frequency and $\sigma$ the electric conductivity.

For the 2D1D model the ansatz

$$
\mathbf{A}=\left(\begin{array}{c}
A_{1,1}(x, y) \phi_{1}(z) \\
A_{1,2}(x, y) \phi_{1}(z) \\
0
\end{array}\right)
$$

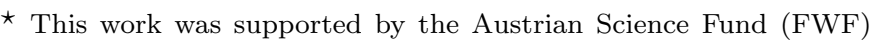
under Project P 27028-N15. is chosen. Here the dependency on the coordinate $z$, aligned with the sheet thickness, is modeled by the linear polynomial function $\phi_{1}$, which is normalized to vary between 1 and -1 along the thickness of the sheet. $A_{1,1}$ and $A_{1,2}$ stand for the two components of one two dimension unknown $\mathbf{A}_{\mathbf{1}}:=\left(A_{1,1}, A_{1,2}\right)^{T} \in H$ (curl). Here and in the following the space $H$ (curl) in two dimensions is defined via the two dimensional curl operator, which is given as

$$
\operatorname{curl} \mathbf{A}_{\mathbf{1}}:=\frac{\partial A_{1,2}}{\partial x}-\frac{\partial A_{1,1}}{\partial y}
$$

To derive the $2 \mathrm{D}$ problem, the ansatz (2) is used in the three dimensional relation (1) for the trial function and the test function, which leads to

$$
\begin{gathered}
\int_{\Omega} \mu^{-1}\left(\begin{array}{c}
-\phi_{1}^{\prime} A_{1,2} \\
\phi_{1}^{\prime} A_{1,1} \\
\operatorname{curl} \mathbf{A}_{\mathbf{1}}
\end{array}\right) \cdot\left(\begin{array}{c}
-\phi_{1}^{\prime} v_{1,2} \\
\phi_{1}^{\prime} v_{1,1} \\
\operatorname{curl} \mathbf{v}_{\mathbf{1}}
\end{array}\right)+ \\
i \omega \sigma\left(\begin{array}{c}
A_{1,1} \phi_{1} \\
A_{1,2} \phi_{1} \\
0
\end{array}\right) \cdot\left(\begin{array}{c}
v_{1,1} \phi_{1} \\
v_{1,2} \phi_{1} \\
0
\end{array}\right) d \Omega=0 .
\end{gathered}
$$

Decomposing the iron sheet $\Omega$ in the form $\Omega=\Omega_{2 D} \times$ $\left[-\frac{d}{2}, \frac{d}{2}\right]$ with the sheet thickness $d$, in (4) the integration over the $z$ coordinate can be carried out, using basic analysis for the integrals involving the known function $\phi_{1}$. This results in the two dimensional problem: Find $\mathbf{A}_{\mathbf{1}} \in H($ curl $)$ so that

$$
\begin{aligned}
& \int_{\Omega_{2 D}} \mu^{-1}\left(\frac{4}{d} \mathbf{A}_{\mathbf{1}} \cdot \mathbf{v}_{\mathbf{1}}+\frac{d}{3} \operatorname{curl} \mathbf{A}_{\mathbf{1}} \operatorname{curl} \mathbf{v}_{\mathbf{1}}\right)+ \\
& i \omega \sigma \frac{d}{3} \mathbf{A}_{\mathbf{1}} \cdot \mathbf{v}_{\mathbf{1}} d \Omega_{2 D}=0
\end{aligned}
$$

for all $\mathbf{v}_{\mathbf{1}} \in H($ curl $)$.

Because it is not straightforward to use physically meaningful boundary conditions in this setting, the problem is driven by first solving a corresponding magnetostatic problem, which is then used as a right hand side for (5).

\section{T FORMULATION}

For the $T$ formulation the three dimensional problem is given as: Find the current vector potential $\mathbf{T} \in H$ (curl) so that

$$
\int_{\Omega} \rho \operatorname{curl} \mathbf{T} \cdot \operatorname{curl} \mathbf{v}+i \omega \mu \mathbf{T} \cdot \mathbf{v} d \Omega=0
$$




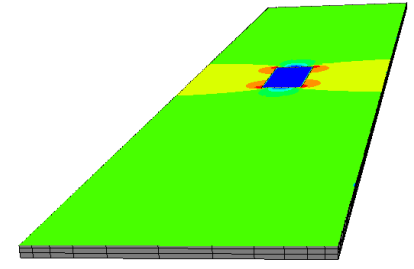

Fig. 1. The iron sheet in the numerical example. Its dimensions are a width of $6 \mathrm{~mm}$, a length of $30 \mathrm{~mm}$ and a thickness of $0.5 \mathrm{~mm}$. At the center of the sheet there is a hole of dimension $1.2 \mathrm{~mm}$ times $3 \mathrm{~mm}$. The material parameters are given as $\mu=1000 \mu_{0}$ and $\sigma=2.08 \times 10^{6} \mathrm{~S} / \mathrm{m}$.

for all test functions $\mathbf{v} \in H$ (curl), with given Dirichlet boundary conditions for $\mathbf{T}$. Here $\rho=\sigma^{-1}$ denotes the electric resistivity.

For the 2D1D model, a similar ansatz as in the case of the A formulation is chosen:

$$
\mathbf{T}=\left(\begin{array}{c}
T_{2,1}(x, y) \phi_{2}(z) \\
T_{2,2}(x, y) \phi_{2}(z) \\
0
\end{array}\right)
$$

Here the behavior in the direction of the thickness is modeled using the even function $\phi_{2}$, which is a quadratic polynomial in $z$.

Analogous to the process for the $A$ formulation, the ansatz (7) is plugged into (6) and the integration over the $z$ direction is carried out analytically, leading to the problem: Find $\mathbf{T}_{\mathbf{2}} \in H(\mathrm{curl})$ so that

$$
\begin{aligned}
& \int_{\Omega_{2 D}} \mu^{-1}\left(\frac{16}{3 d} \mathbf{T}_{\mathbf{2}} \cdot \mathbf{v}_{\mathbf{2}}+\frac{8 d}{15} \operatorname{curl} \mathbf{T}_{\mathbf{2}} \operatorname{curl} \mathbf{v}_{\mathbf{2}}\right)+ \\
& i \omega \sigma \frac{8 d}{15} \mathbf{T}_{\mathbf{2}} \cdot \mathbf{v}_{\mathbf{2}} d \Omega_{2 D}=0 .
\end{aligned}
$$

for all $\mathbf{v}_{\mathbf{2}} \in H$ (curl). The problem is again driven using the solution of an auxiliary problem for the right hand side.

\section{A NUMERICAL EXAMPLE}

In order to test the models developed in sections 2 and 3 , a simple numerical example is carried out. The dimensions of the problem and the used material parameters can be taken from figure 1 .

Figure 2 shows the relative error in the calculated losses. The reference solution was calculated by solving the original problems, (1) and (6), on a three dimensional mesh, respectively. It can be seen that the error increases with higher frequencies, as expected. Out of the given 2D1D models, the one for the $T$ formulation performs better, because it is able to simulate the boundary effects, as can be seen in figures 3 and 4 .

\section{CONCLUSION}

The presented method allow for a reasonably precise calculation of the eddy current losses for low frequencies. An extension into a higher frequency range is possible by including additional ansatz functions. Future work will

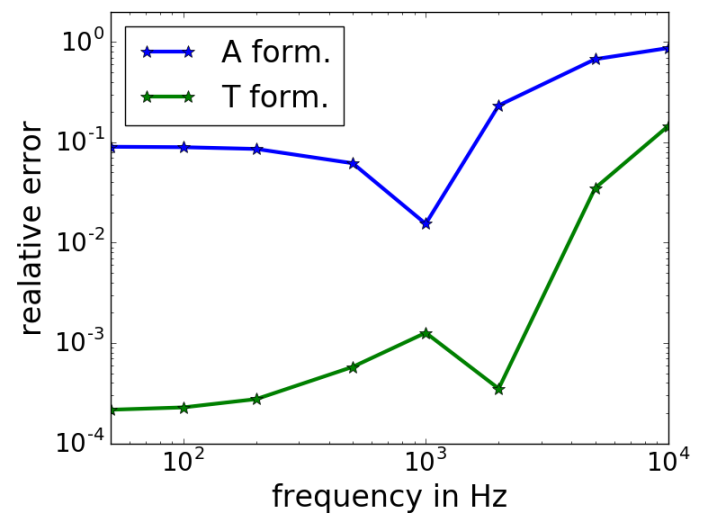

Fig. 2. The relative error in the calculated losses for both formulations.

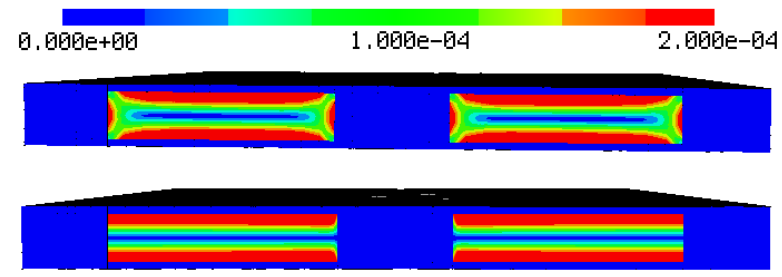

Fig. 3. Absolute value of the magnetic vector potential $A$ in a cross section of the sheet for the reference solution (top) and the 2D1D model (bottom) at 100Hz.

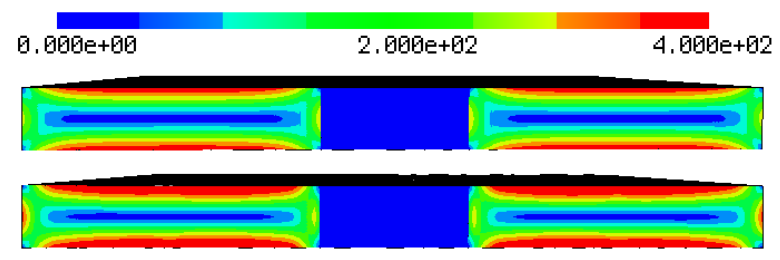

Fig. 4. Absolute value of $\operatorname{curl} T$ in a cross section of the sheet for the reference solution (top) and the 2D1D model (bottom) at $100 \mathrm{~Hz}$.

include testing the applicability of these models in the nonlinear setting. An additional extension will be the development of a modification for the $A$ formulation, which is able to resolve the boundary effects.

\section{REFERENCES}

Bottauscio, O. and Chiampi, M. (2002). Analysis of laminated cores through a directly coupled 2-d/1-d electromagnetic field formulation. IEEE Trans. Magn., 38, 2358-2360.

F. Henrotte, S. Steentjes, K.H. and Geuzaine, C. (2015). Pragmatic two-step homogenisation technique for ferromagnetic laminated cores. IET Sci. Meas. Technol., 9, 152-159.

Hollaus, K. and Schöberl, J. (to be published). Some two-dimensional multiscale finite element formulations for the eddy current problem in iron laminates. IEEE Trans. Magn., to be published.

J. Pippuri, A. Belahcen, E.D. and Arkkio, A. (2010). Inclusions of eddy currents in laminations in two-dimensional finite element analysis. IEEE Trans. Magn., 46, 29152918. 